\title{
Comparative Study of the Adsorption of Thiol and Isocyanide Molecules on Silver Surface by In Situ Surface-Enhanced Raman Scattering
}

Jaewan Ahn, Shi Shi, Bonnie Vannatter, and Dong Qin*

School of Materials Science and Engineering, Georgia Institute of Technology, Atlanta, Georgia 30332, United States

*Corresponding author: dong.qin@mse.gatech.edu 


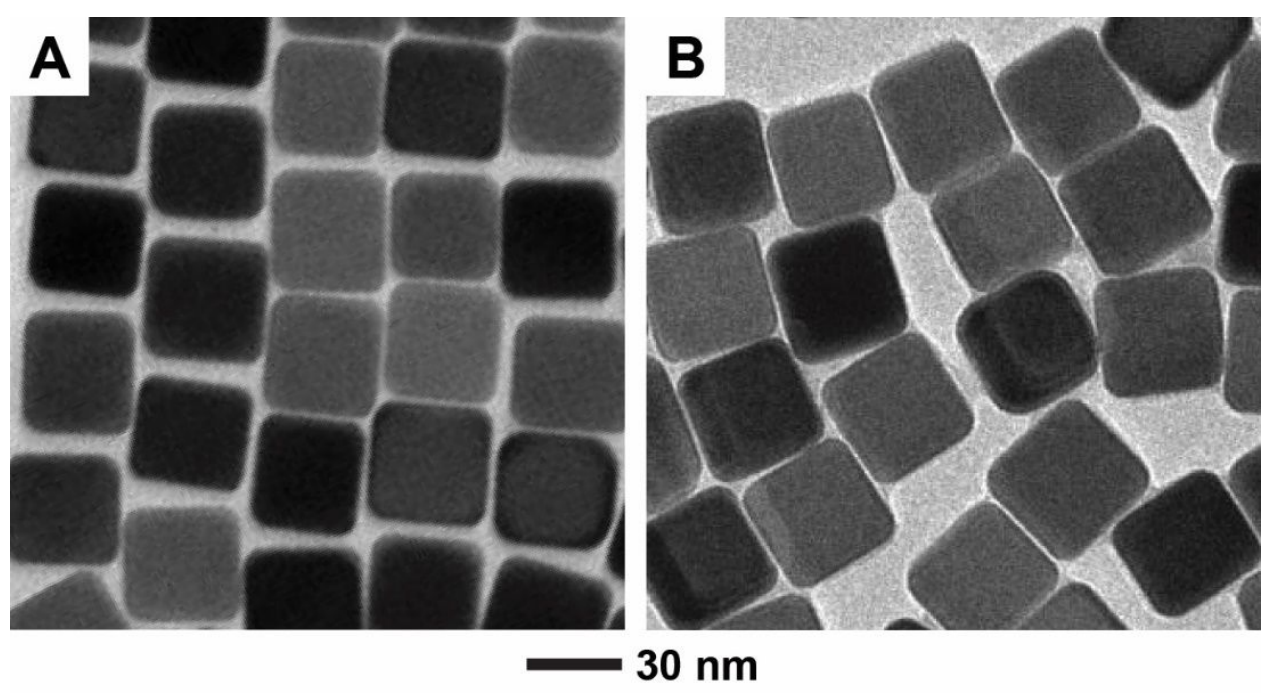

Figure S1. TEM images of (A) the as-synthesized Ag nanocubes and (B) the acid-treated Ag nanocubes. 


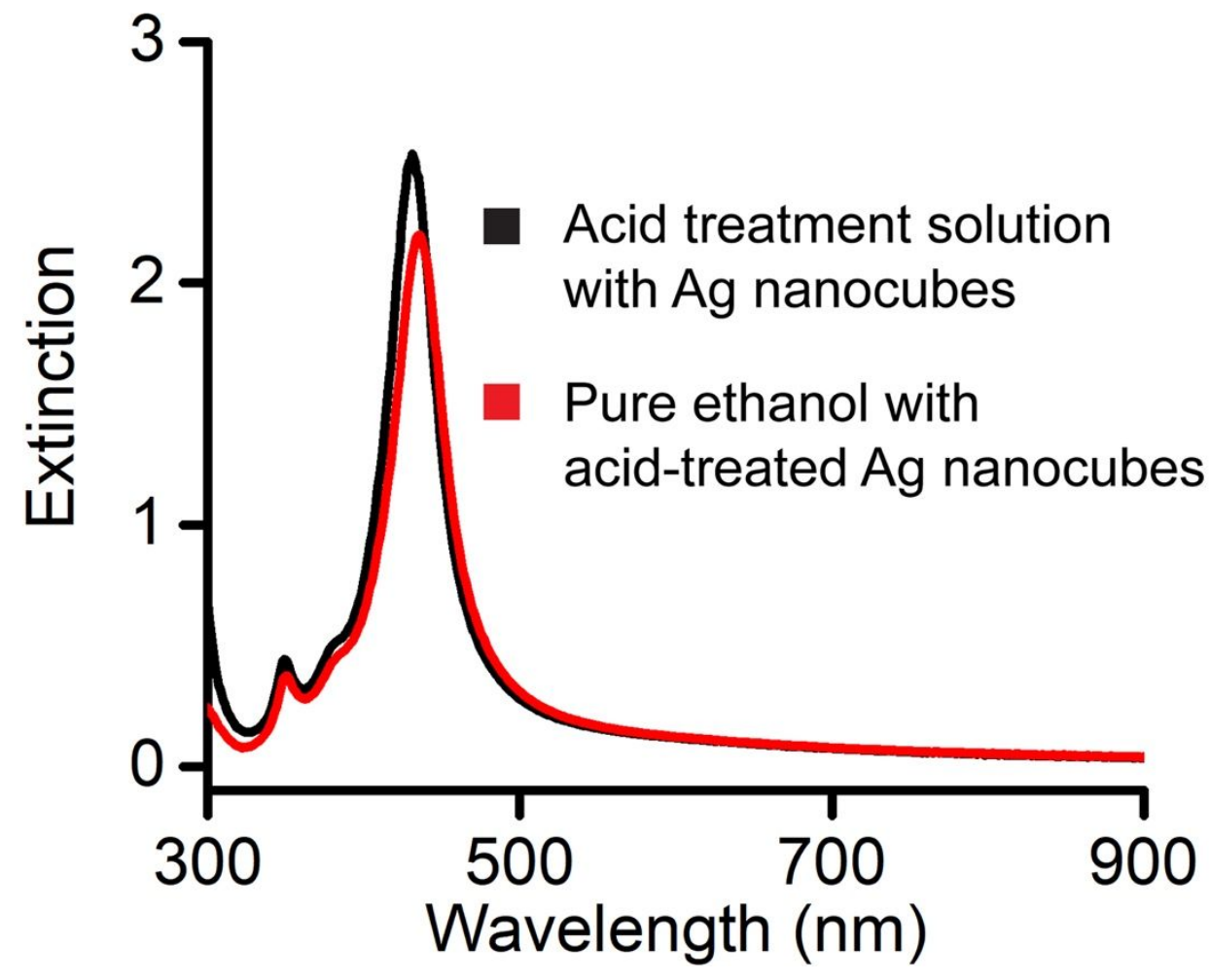

Figure S2. UV-vis spectra of Ag nanocubes dispersed in an acid treatment solution (black curve) and the acid-treated Ag nanocubes re-dispersed in pure ethanol (red curve). 

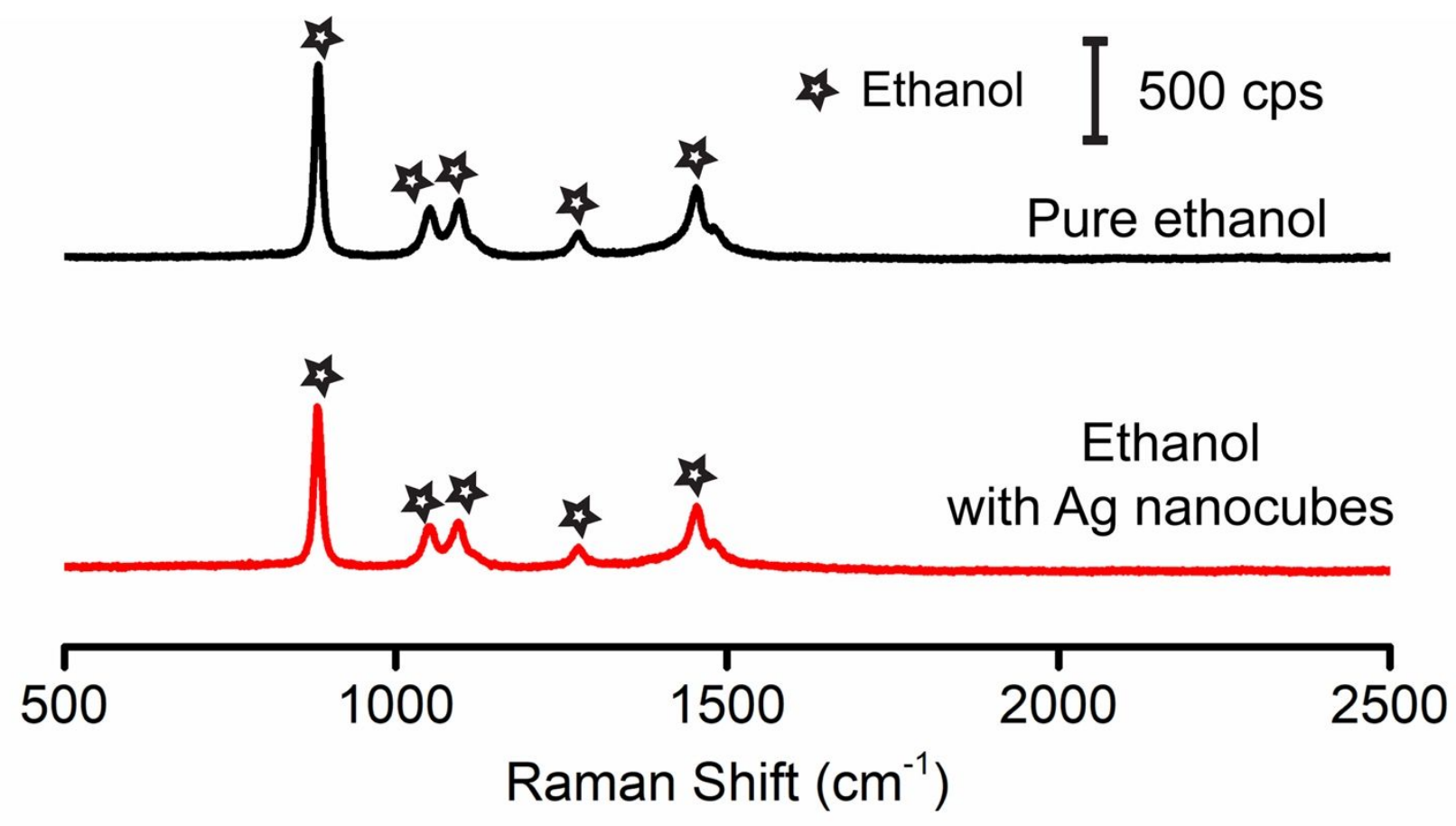

Figure S3. Raman spectra recorded from pure ethanol (black) and ethanol containing as-prepared Ag nanocubes (red). 


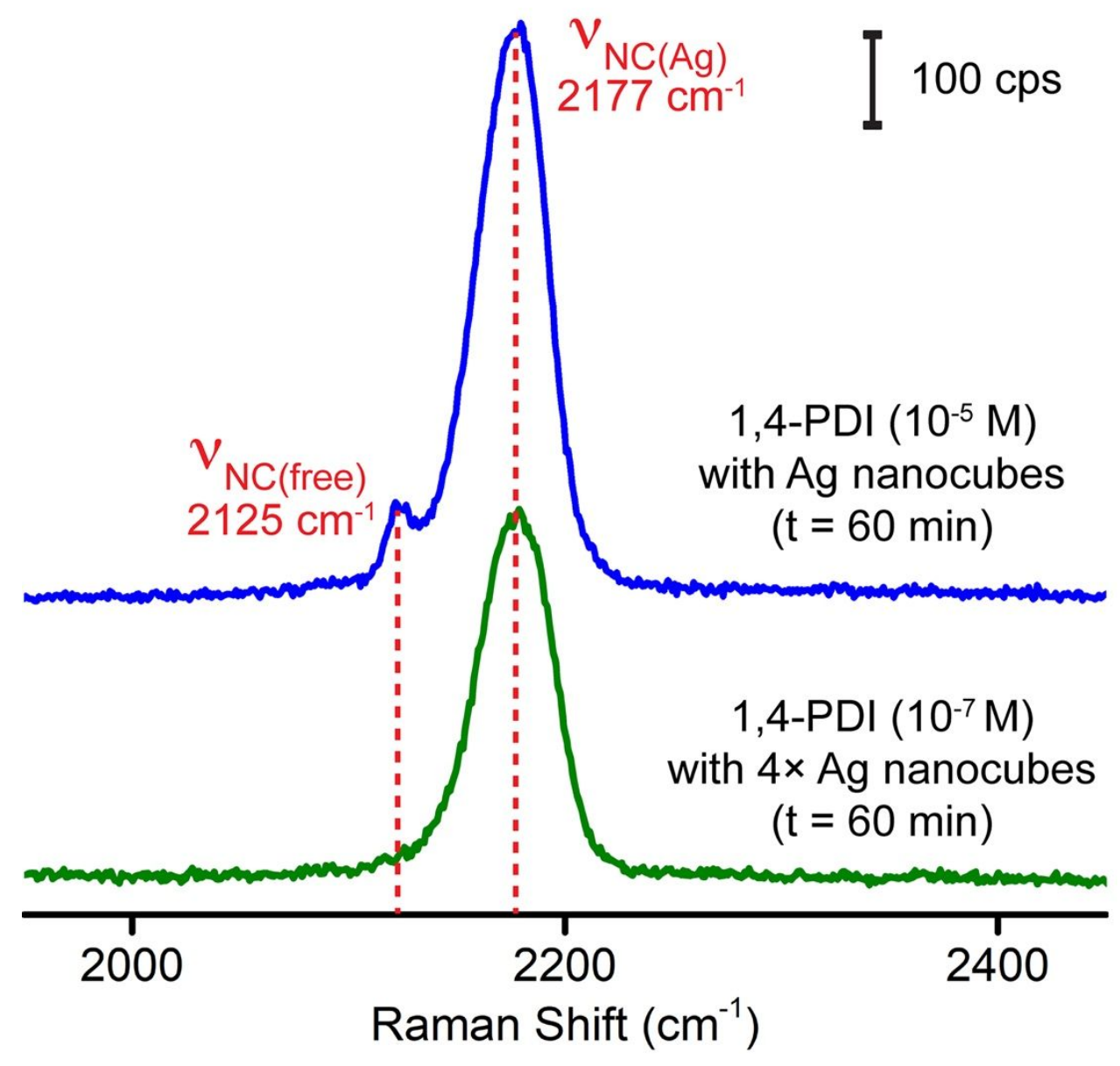

Figure S4. The SERS spectra of $1,4-\mathrm{PDI}$ collected at $t=60 \mathrm{~min}$ by using (A) Ag nanocubes prepared by 1,4-PDI ethanol solutions at $10^{-5} \mathrm{M}$ and (B) $\mathrm{Ag}$ nanocubes prepared by 1,4-PDI ethanol solutions at $10^{-7} \mathrm{M}$ with the number of particles quadrupled from those in (A). 
Table S1. Raman data of the characteristic peak of Si as periodically measured throughout all the SERS experiments.

\begin{tabular}{c|ccc}
\hline$\#$ & Centre & Width & Height \\
\hline 1 & 520.35 & 5.11 & 110652 \\
2 & 520.38 & 4.97 & 110645 \\
3 & 520.35 & 5.02 & 115457 \\
4 & 520.26 & 5.02 & 108978 \\
5 & 520.25 & 5.03 & 110601 \\
6 & 520.20 & 5.30 & 108247 \\
7 & 520.32 & 4.87 & 107954 \\
8 & 520.39 & 5.24 & 112402 \\
9 & 520.29 & 5.27 & 108795 \\
10 & 520.10 & 5.09 & 112784 \\
11 & 520.34 & 5.54 & 115776 \\
12 & 520.44 & 5.07 & 117896 \\
13 & 520.22 & 5.42 & 119255 \\
14 & 520.10 & 5.09 & 112784 \\
15 & 520.32 & 4.87 & 122134 \\
16 & 520.32 & 5.48 & 117520 \\
17 & 520.32 & 5.16 & 116003 \\
18 & 520.31 & 5.42 & 113424 \\
19 & 520.37 & 5.11 & 118993 \\
20 & 520.19 & 5.61 & 106963 \\
\hline Mean & $\mathbf{5 2 0 . 2 9}$ & $\mathbf{5 . 1 9}$ & $\mathbf{1 1 3 3 6 3}$ \\
\hline Standard & $\mathbf{0 . 0 9}$ & $\mathbf{0 . 2 2}$ & $\mathbf{4 3 4 6}$ \\
Deviation & & & \\
\hline & & & \\
\hline
\end{tabular}

